\title{
Percepción de una Formación Académica enfocada a la Investigación Cientifica constante en la Universidad Nacional Mayor de San Marcosi
}

\section{Perception of an Academic Education focused on constant Scientific Researchat the National University of San Marcos}

\author{
Neisser Pino Romero ${ }^{2}$ \\ Brisaida Isabel Flores Barrenechea ${ }^{3}$
}

\section{RESUMEN}

En presente artículo de investigación intenta recolectar la percepción que se tiene de la investigación científica en la formación académica de los estudiantes universitarios, la cual es la puerta hacia la investigación científica continua que se debe realizar para buscar una innovación de conocimientos y métodos científicos, técnicas y perspectivas que relacionen la ciencia y la sociedad. La investigación científica reflejada en artículos científicos desde la formación de pregrado, y con más énfasis en la formación de posgrado deben ser el sustento académico para el desarrollo del

\begin{abstract}
1 El presente trabajo busca analizar la percepción ante un enfoque de investigación en la formación académica de pregrado mediante la publicación de artículos de investigación en revistas científicas.

2 Docente de la Facultad de Ciencias Económicas de la Universidad Nacional Mayor de San Marcos. Docente de la Facultad de Ciencias y Filosofía "Alberto Cazorla Talleri" de la Universidad Peruana Cayetano Heredia. Investigador Visitante en Carleton University (Canada's Capital University). Magíster en Matemática Aplicada con mención en Matemática Computacional (UNMSM). Licenciado en Computación Científica (UNMSM).Email: neisser.pino@unmsm.edu.pe,neisser.pino@upch.pe 3 Médico Cirujano de la Universidad Amistad de los Pueblos (Rusia). Especialidad en Medicina Nuclear en la Universidad Nacional Mayor de San Marcos. Maestrista en Salud Pública en mención Gestión Hospitalaria en la Universidad Nacional Federico Villareal. Email: brisaidaf@gmail.com
\end{abstract}

(C) Los autores. Este artículo es publicado por Pensamiento Crítico de la Facultad de Ciencias Económicas, Universidad Nacional Mayor de San Marcos. Este es un artículo de acceso abierto, distribuido bajo los términos de la licencia Creative Commons Atribucion - No Comercia_Compartir Igual 4.0 Internacional. (http:// creativecommons.org/licenses/by-nc-sa/4.0/) que permite el uso no comercial, distribución y reproducción en cualquier medio, siempre que la obra original sea debidamente citada. 
país. Por lo cual, cuán importante es la investigación científica que se debe realizar para lograr una mejor educación en la sociedad.

Palabras Clave: Formación académica; investigación científica; artículos científicos

\section{ABSTRACT}

In this research article, the aim is to collect the perception of scientific research in the academic education of university students, which is the door to continuous scientific research that must be carried out in order to seek an innovation of scientific and technical knowledge and methods and perspectives that relate science and society. The scientific research reflected in scientific articles from undergraduate training, and with more emphasis on postgraduate training should be the academic support for the development of the country. Therefore, how important is the scientific research that must be done to achieve a better education in society.

Keywords: Academic training; scientific research; scientific articles; scientific innovation.

JEL Classification Codes: I21.

\section{Introducción}

Desde la historia de la humanidad se ha dedicado a investigar sobre su entorno de diferentes formas para poder lograr sus objetivos. La universidad ha sido una institución educativa donde se iba formando las ideas y la creación de conocimientos tanto divulgativos como los métodos intuitivos en las diversas áreas de la ciencia. Quizás en la evidencia más concreta donde la ciencia se iba formando era mediante los conflictos bélicos entre los diferentes pueblos y a través de la historia se ha ido generando conocimiento y tecnología para los distintos problemas y escenarios donde la sociedad necesitaba innovación para continuar con las perspectivas de creación de nuevos métodos científicos para dar luces en medio de la obscuridad.

Según Roger Guerra (1979), los diferentes países han ido generando conocimientos y tecnologías para su desarrollo como país, y de manera esencial para la sociedad, mejorando la calidad de vida de los sus habitantes. Pero este desarrollo va de la mano con la educación y la investigación científica de una manera constante y continua. Los países que invierten una gran cantidad de dinero en la educación y de manera particular en la investigación tienden a generar conocimientos propios que pueden ser utilizados para el mejoramiento y perfeccionamiento de diversos métodos que son utilizados en el día a día. La investigación 
científica en el transcurso de la formación académica ha ido decayendo con el pasar de los años desde una perspectiva global del país, pero también hay que considerar que ha habido pequeños incrementos en las ciencias básicas. Detalles de cómo la investigación científica se va generando de manera más robusta y significativa en la capital es el detalle que hay un centralismo de la educación superior para la investigación, por mientras que en los demás departamentos este crecimiento es casi nulo, estos puntos importantes sobre la historia de la investigación científica del país en los últimos años lo manifiestan dos artículos periodísticos de Sandro Medina (2015) del Comercio y de la redacción de Gestión (2014).

Quizás en estos últimos años, el tema de la investigación científica en la mayoría de las carreras profesionales que se imparten en las universidades peruanas es un enfoque importante de la nueva ley universitaria. Por lo cual, el presente artículo se centrará como la investigación científica es percibida en estudiantes y egresados de dos universidades peruanas (Universidad Nacional Mayor San Marcos y Universidad Peruana Cayetano Heredia) donde quizás la exigencia de realizar investigación y de publicarla en revistas científicas es el punto esencial del presente trabajo de investigación. Se tendrá un enfoque más detallado a la universidad pública de manera más particular a la facultad de ciencias económicas.

\section{La ley universitaria y la investigación científica}

La ley universitaria que se ha promulgado el julio del 2014, comenzó a dar un nuevo inicio a la formación académica en todas las universidades peruanas debido que ahora las universidades deben alinearse a la perspectiva de la nueva ley, donde la ley universitaria ha tenido aciertos, errores y omisiones es lo que expresa Mario Mejía (2014). Donde se enunciará una parte del artículo 3 de la presente ley: La universidad es una comunidad académica orientada a la investigación y a la docencia, que brinda una formación humanística, científica y tecnológica con una clara conciencia de nuestro país como realidad multicultural. Adopta el concepto de educación como derecho fundamental y servicio público esencial. De acuerdo a la definición que brinda la ley universitaria se permite un enfoque primordial a la investigación científica en todas las carreras profesionales que brinda la universidad, debido que tiene una percepción que la investigación es la generadora de nuevos conocimientos para el beneficio de la sociedad como lo manifiesta el artículo 6 de la ley del capítulo I. 
De acuerdo al capítulo IV de la ley nos expresa una importancia sobre la investigación. En el artículo 48 expresa:

La investigación constituye una función esencial y obligatoria de la universidad, que la fomenta y realiza, respondiendo a través de la producción de conocimiento y desarrollo de tecnologías a las necesidades de la sociedad, con especial énfasis en la realidad nacional. Los docentes, estudiantes y graduados participan en la actividad investigadora en su propia institución o en redes de investigación nacional o internacional, creadas por las instituciones universitarias públicas o privadas.

En este capítulo se vuelve a considerar los lineamientos que puede corregirse en la ley universitaria en contraste con la realidad del país y de sus comunidades académicas. Según Mejía (2014) expresa

La nueva ley universitaria ha generado encontradas opiniones. Algunos especialistas en temas educativos consultados por El Comercio resaltan que la norma tiene algunos aspectos buenos que mejorarán la calidad del sistema universitario, pero también resaltan otros que deben corregirse y mejorarse, como, por ejemplo, no dar importancia a la educación virtual y solo toman en cuenta la presencial.

Siendo un contraste entre la producción científica orientada a la investigación o una difusión académica de una manera masiva. Estas perspectivas están en debate para dilucidar quizás una mejor orientación de la formación académica a nivel universitario.

Como se puede ver la ley universitaria intenta brindar unos nuevos senderos de la formación universitaria en todo el país con un enfoque de investigación científica en todo momento para la creación de nuevos conocimientos para el uso de la comunidad y de la sociedad. Pero se debe tener en cuenta muchos detalles cuando se desea realizar investigación científica que pueda ser reconocida como tal. La formación académica de los estudiantes que apoyan a los docentes a realizar investigación, y como los docentes apoyan a los estudiantes a realizar investigación es el complemento idóneo para una adecuada formación científica a través del tiempo. Esto va mostrando en los estudios realizados en el artículo "Actitudes hacia la investigación científica en docentes de metodología de la investigación" que presentaron Aldana y Joya (2011).

Del mismo modo, Barahona y Medina (2015) expresan en su artículo "Percepción de los docentes y estudiantes hacia el proceso enseñanzaaprendizaje de la investigación en la Facultad de Ingeniería, Ciudad 
Universitaria, 2014". Donde veían esta importancia de enfocar de una manera significativa la investigación en la formación académica. Y como complemento también lo expresa Coraggio (1996) que

Plantea que se debe motivar a los estudiantes, desde el ejemplo y la reflexión, para que asuman actitudes y valores asociados al trabajo científico y hermenéutico, que son los que acompañan al tipo ideal de profesional o científico. Sin embargo, en el docente mismo no se evidencian acciones propias del quehacer investigativo, como preguntar significativamente, leer comprensivamente, escribir, diseñar y desarrollar proyectos de investigación (Brunner, 1997; Serrano, 1997).

Para Desantes-Guanter y López (1996)

el investigador ha de estar dispuesto al esfuerzo intelectual, a la incertidumbre, a la prudencia y a potenciar valores como la honestidad, la curiosidad, el pensamiento crítico, el trabajo sistemático y ético, así como la admisión de la transitoriedad del conocimiento. Valores que deben ser extensivos a los docentes de investigación en tanto que en la mayoría de casos el docente de investigación está vinculado laboralmente como investigador.

Como se va observando en los diferentes países que han considerado la realización de investigación como un eje fundamental en su formación académica han tenido un proceso de adaptación para ir obteniendo los logros académicos que brinda la investigación científica.

\section{Percepción de la formación académica de la universidad orientado a la investigación}

La investigación científica es algo más amplio, por no decir que es un mundo lleno de tantas reglas, maneras, enfoques y formas de realizar investigación en cada una de las áreas de la ciencia. Como se ha mencionado en la Universidad Nacional Mayor de San Marcos (UNMSM) distribuye todas sus escuelas académicas profesionales (carreras profesionales) en áreas respectivas a su enfoque científico. Según la Oficina Central de Admisión (OCA) de la universidad se distribuye de la siguiente manera:
A. Ciencia de la Salud
B. Ciencias Básicas
C. Ingenierías
D. Ciencias Económicas y de la Gestión
E. Humanidades, y Ciencias Jurídicas y Sociales 
Se puede considerar que todas las carreras que se brindan en todas las universidades peruanas (públicas y privadas) se pueden clasificar en estas cinco áreas mencionadas. Estas referencias consideradas de la clasificación de las carreras es la más adecuada debido que la distribución de los enfoques de investigación de acuerdo a la carrera y del área respectiva necesita un lineamiento especializado para una idónea formación científica. La universidad que en sus pilares está el cimiento de la investigación para un continuo proceso de investigación conjunta entre docentes y estudiantes durante la estancia en la universidad, y luego al graduarse y comenzar a ejercer la profesión aún se mantenga la investigación buscando mejorar los procesos existentes e innovar nuevos procesos para mejorar la sociedad. Esta concepción la tienen los países que se le denominan países de primer nivel o países desarrollados, debido que siempre están en constante renovación y mejoramiento. Es una tradición que se forma desde el primer año de formación, y un sendero que se vislumbra en los años anteriores de formación académica en la escuela. Los ingleses tenía una frase ejemplar sobre su visión de sociedad: La tradición nos define.

Luego, en el artículo de Bernecker titulado "La investigación histórica del tiempo presente en Alemania" y la redacción de Nuclear Physics Experience (NUPEX), comentan que Alemania seguiría cimentando la investigación científica quizás de un enfoque bélico e inversionista donde se vería en la segunda guerra mundial; no se quiere tocar el enfoque moral ni ético de la segunda guerra mundial sino de cómo la ciencia, la tecnología y la innovación puede ayudar a mejorar un país. Para terminar esta sección solamente quedaría decir que la formación académica universitaria debería ser una tradición que debe definir la formación y la visión del porque se forma uno en una universidad.

Los diferentes países con el paso del tiempo han ido concretándose en la línea de investigación que deben tener sus profesionales porque cada país tiene sus propios problemas que resolver, sus propios conflictos que solucionar y sus propios dilemas que dilucidar, y este detalle contempla la nueva ley universitaria con respecto a la universidad, es como lo enuncia la redacción de Portal Alemania (2016), y también expresa. 
Alemania es uno de los estados con mayor nivel en el ámbito de la investigación, prueba de ello son los numerosos centros de investigaciones excelentes de gran prestigio o el hecho de que Alemania es el país europeo que más patentes registra con cerca de 6.200 patentes de un total de 52.000 en todo el mundo y junto con EE.UU. y Japón uno de los tres países líderes en innovación a nivel mundial.

\section{Percepción de los estudiantes y egresados sobre la perspectiva de una formación académica orientado a una investigación continua.}

En esta sección se dará un enfoque diferente que la nueva ley universitaria indica; por lo cual se ha realizado una encuesta de opinión pública con respecto a las directrices que se propondrá con respecto a la formación académica orientado a una investigación científica constante. La presente perspectiva sobre la investigación tiene quizás una posible consecuencia de la ley universitaria.

El presente trabajo de investigación es netamente descriptivo que se ha generado sobre una opinión con respecto a una posibilidad de cambiar el entorno académico profesional en todo el país, bajo la índole de que la preparación, la formación y la instrucción de las universidades deben resolver del propio país buscando nuevas tecnologías y formas de optimizar los procesos de tal modo que puedan ser eficientes y de alta calidad. La innovación siempre tiene factores favorables y desfavorables que se tienen que superar por el bien de la sociedad.

Las decisiones que buscan nuevos horizontes pueden ser no aceptados en un inicio por el miedo quizás a un sendero desconocido, difícil, innecesario, complicado; quizás sean las palabras que muchas personas han pensado antes de empezar en un requisito que se pueda imponer de un momento a otro. Con estas últimas palabras se terminara el pequeñísimo preámbulo de un sendero en la investigación bajo la percepción de estudiantes y profesionales. La encuesta utilizada para el presente trabajo consistió en un total de 14 preguntas de elección múltiple, donde eran preguntas de alternativa múltiple y se realizó únicamente vía virtual mediante la aplicación de los Formularios de Google (https:// www.google.com/intl/es-419_pe/forms/about/) para facilitarnos el análisis descriptivo de los resultados. 
Las preguntas de la encuesta se enfocan en el acuerdo o desacuerdo de ciertos criterios ya establecidos actualmente por la ley universitaria, como también por posibles consideraciones ante la perspectiva propuesta en el presente artículo. De las preguntas realizadas en la encuesta se podría dividir en dos perspectivas: la primera perspectiva propuesta por la nueva ley universitaria y el propuesto en el presente trabajo como una alternativa para una constante investigación en la realización profesional. Solamente se tendrá en cuenta en la formación de pregrado debido que esta formación es de manera obligatoria para la obtención del título profesional por mientras que la formación de posgrado es de manera optativa de los profesionales.

En la primera pregunta realizada en la encuesta es la situación en que muchos estudiantes podrían estar de acuerdo o desacuerdo de manera personal, pero la ley universitaria indica en el artículo 45 con respecto a la obtención de grados y títulos. De manera particular en el sub-artículo 45.1 con respecto al grado de bachiller se expresa: requiere haber aprobado los estudios de pregrado, así como la aprobación de un trabajo de investigación y el conocimiento de un idioma extranjero, de preferencia inglés o lengua nativa. Y el sub-articulo 45.2 con respecto al título profesional expresa: requiere del grado de Bachiller y la aprobación de una tesis o trabajo de suficiencia profesional. Las universidades acreditadas pueden establecer modalidades adicionales a estas últimas. El título profesional solo se puede obtener en la universidad en la cual se haya obtenido el grado de bachiller.

De estas consideraciones nos centraremos en la realización de una tesis de investigación para obtener el grado de bachiller y el título profesional (trabajo de investigación para el bachillerato y tesis para el título profesional). Bajo esta perspectiva se realizó la pregunta: ¿Estás de acuerdo con realizar dos tesis para obtener el grado de Bachiller y el título profesional, respectivamente?

Lo cual las respuestas obtenidas están representado en la siguiente grafica teniendo en cuenta una base de 1000 encuestados con respecto a la nueva disposición de la nueva ley universitaria donde a diferencia de la anterior, la obtención del grado académico de bachiller era automático y para obtener el título profesional se tenía que realizar una tesis (se está descartando la opción de las otras modalidades). 
De la pregunta anterior se obtiene prácticamente un rechazo a la nueva normativa que exige la ley universitaria con un $46.9 \%$ por mientras que un $14.2 \%$ se mantienen en una duda si apoyar la medida establecida por la ley universitaria. Esta pregunta nos brinda obtener información preliminar con respecto a las preguntas siguientes que nos permitirán obtener más lineamientos sobre la percepción de una propuesta de una formación académica orientada a la investigación.

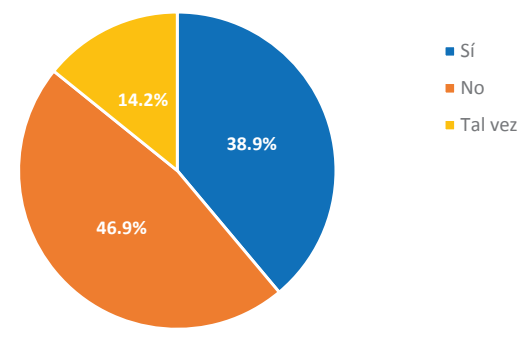

Gráfico 1. Conformidad ante la realización de dos tesis para obtener el grado de bachiller y el título profesional, respectivamente.

Fuente: Elaboración propia.

Por otro lado, la nueva exigencia que brinda la ley universitaria para poder ejercer la docencia a nivel de pregrado se expresa en el subartículo 82.1 es la siguiente: para el ejercicio de la docencia universitaria, como docente ordinario y contratado es obligatorio poseer el grado de nivel de maestro. Este requerimiento se está exigiendo desde que se promulgó la ley universitaria para el ejercicio de la docencia, de manera particular la Universidad Nacional Mayor de San Marcos. Con respecto a esta consideración de los docentes universitarios se realizó la siguiente pregunta: ¿Crees que el grado de maestro (como mínimo) de los docentes incrementará el nivel académico de la formación universitaria?

Realmente hay una mayoría que apoya la medida de exigir el grado de maestro para ejercer la docencia universitaria. Y un $69.2 \%$ apoya esta medida que equivale a 692 encuestados. Por mientras, que un $30.8 \%$ no está de acuerdo con la medida exigida, debido que se tiene que el tener un grado de maestro no es algo imprescindible para poder brindar una educación de calidad. 


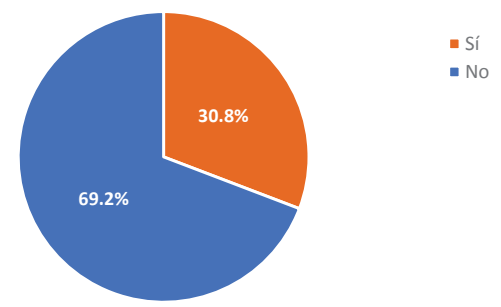

Gráfico 2. Nivel de conformidad ante el requisito obligatorio de la maestría para los docentes universitarios.

Fuente: Elaboración propia.

Con respecto a esta perspectiva, se preguntó una interrogante relacionada a la formación académica que puede brindar un docente. Y siempre cuando recibe clases de algún curso siempre busca que el docente tenga un buen nivel académico pero a la vez que tenga un buen nivel pedagógico, y esta interrogante es clave en la elección de docentes para los estudiantes que buscan aprender conocimientos de alto nivel pero que pueden comprenderlos de una manera accesible mediante la didáctica del docente. Pero no siempre se encuentra un adecuado complemento entre el nivel académico y el nivel pedagógico en los docentes; por lo cual se planteó una posición extrema entre la elección de uno solo, de esta manera se presentó la siguiente pregunta: ¿Cuál crees que es más importante para la formación universitaria, el nivel académico o el nivel pedagógico del docente?

En relación a la decisión de los encuestados (estudiantes y profesionales) se sitúa de una manera casi igual, debido que la diferencia es de 5.2\% (mínimo). Pero se ha considerado que el nivel académico predomina en la elección de los encuestados, quizás porque los conocimientos, la experiencia, la innovación se refleja en el nivel académico más que el nivel pedagógico, lo que no se quiere decir que el nivel pedagógico sea de menor importancia que el nivel académico de un docente. 




Gráfico 3. Elección ante la preferencia del nivel académico o el nivel pedagógico en un docente universitario.

Fuente: Elaboración propia.

Ahora que se realizado una pequeña descripción de la opinión de los encuestados sobre las directrices que brinda la actual ley universitaria, se pasará a analizar las preguntas que se enfocan en la perspectiva de una investigación constante de cada una de las profesiones que se imparten en las universidades peruanas (públicas y privadas). Las preguntas se enfocaron donde la investigación es el centro de la formación académica, buscando generar conocimiento e innovar en la medida que sea posible, lo que la actual ley universitaria indica en sus artículos sobre los fines de la universidad.

La segunda perspectiva dada en la encuesta del enfoque presentado en el artículo presentara una exigencia por sí misma, intrínseca en la formación académica. Esto es, debido que en la misma ley universitaria plantea un artículo específico para los docente investigadores, sin tener en consideración que investigación lo pueden realizar todos los estudiantes y los profesionales. El artículo 86 expresa:

el docente investigador es aquel que se dedica a la generación de conocimiento e innovación, a través de la investigación. Es designado en razón de su excelencia académica. Su carga lectiva será de un curso por año. Tiene una bonificación especial del cincuenta por ciento (50\%) de sus haberes totales. Está sujeto al régimen especial que la universidad determine en cada caso. El Vicerrectorado de Investigación o la autoridad competente evalúa cada dos años, la producción de los docentes, para su permanencia como investigador; en el marco de los estándares del Sistema Nacional de Ciencia, Tecnología e Innovación Tecnológica (SINACYT). 
El presente artículo está enfocado únicamente para los docentes universitarios que sean considerados docentes investigadores. No menciona nada sobre los estudiantes o profesionales que realicen investigación dentro de la universidad o fuera de ella. Por lo cual, se presentará diversas preguntas sobre la concepción de investigación a estudiantes y profesionales. Antes de mostrar y analizar los respectivos resultados se presentará algunos detalles sobre los estudiantes y profesionales encuestados que nos permitan tener una mejor perspectiva y análisis de las opiniones y conocimientos sobre realizar investigación, pero sobretodo en la redacción de artículos científicos publicadas en revistas científicas indexadas o no indexadas.

Como se había mencionado, se ha realizado una base de datos de 1000 encuestados para el presente trabajo que se realizó en un periodo de cinco meses (junio 2017 a octubre 2017), donde se ha encontrado dos intervalos marcados con respecto a las edades. Con respecto a los estudiantes se tiene un intervalo entre los 19 y 22 años de edad, por mientras que el otro intervalo correspondiente a los profesionales se sitúa entre los 30 y 35 años de edad. Y otro detalle significativo que se había mencionado con respecto al estudio de campo, es que se tiene encuestados de dos universidades: Universidad Nacional Mayor de San Marcos (universidad pública) que posee el $63 \%$ de los encuestados y la Universidad Peruana Cayetano Heredia (universidad privada) que posee el $37 \%$ de los encuestados. Debido que la formación académica no distingue si proviene de una universidad pública o privada. Pero es bueno tener en cuenta la concepción que se tiene con respecto a la investigación.

Y es por eso que se ha considerado estas dos universidades, Universidad Nacional Mayor de San Marcos se posiciona en el cuarto puesto a nivel nacional, pero en el primer puesto a nivel de las universidades nacionales, por mientras que la Universidad Peruana Cayetano Heredia se posiciona en el segundo puesto a nivel nacional y a nivel de las universidades privadas, según la revista América Economía en el artículo de Carlos Alcántara y Andrés Almeida, y de aquí el periódico La Republica lo expresa de manera puntual en cuanto a los resultados.

Y como anteriormente se había mencionado sobre las áreas de las carreras profesionales que brinda las universidades se podría reunir en 
cinco áreas según la OCA de la Universidad Nacional Mayor de San Marcos, de las cuales se tiene el siguiente resumen de los encuestados en su totalidad.
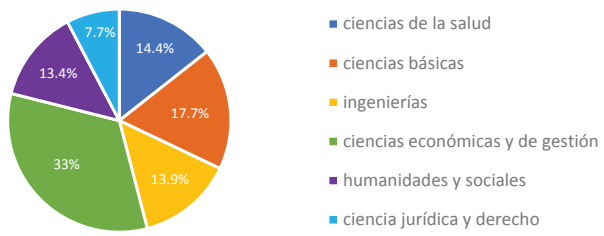

Gráfico 4. Distribución de los encuestados en las áreas académicas.

Fuente: Elaboración propia.

De la presente gráfica se puede apreciar cómo se ha distribuido las diferentes áreas de las carreras profesionales de los encuestados. De manera particular, nos enfocaremos en el área de ciencias económicas y de gestión, debido que se quiere detallar de una manera específica a los encuestados (estudiantes y profesionales) que pertenecen a la facultad de ciencias económicas de la Universidad Nacional Mayor de San Marcos.

La facultad de ciencias económicas de la universidad posee tres escuelas académicos profesionales (carreras profesionales) cuales son: Economía, Economía Publica y Económica Internacional. De donde se ha obtenido un total de 270 encuestadosde la presente facultad de toda la base de datos considerada. Y su respectiva distribución se ha dado de la siguiente manera:

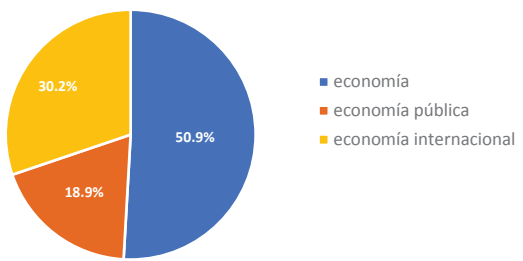

Gráfico 5. Distribución de los encuestados pertenecientes a la Facultad de Ciencias Económicas de la Universidad Nacional Mayor de San Marcos.

Fuente: Elaboración propia. 
Con estos detalles preliminares nos centraremos en las preguntas sobre la investigación en la formación académica. Quizás la primera pregunta de la perspectiva de la investigación, es sobre la importancia de la investigación en sí misma en la formación académica. La pregunta fue la siguiente: ¿Está de acuerdo de realizar investigación en pregrado? Lo cual, la gran mayoría respondió afirmativamente, es decir, un $84.8 \%$ de los encuestados estuvo de acuerdo.

Por lo cual, hay una gran mayoría de encuestados (848 participantes) están a favor de realizar investigación en pregrado, esto indica que una adecuada formación académica permitiría la generación de investigación y por ende la apertura a la publicación de artículos de investigación en revistas científicas.

Las dos siguientes preguntas son complementarias debido que uno conlleva a la otra. Y genera la disyuntiva de cómo se debe orientar la formación académica, los cursos de la malla curricular y la perspectiva académica que el docente debe orientar. La primera pregunta se presentó de la siguiente forma: ¿Crees que la formación académica debe orientarse a la demanda del mercado?

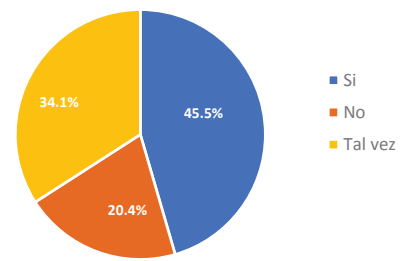

Gráfico 6. Conformidad que formación académica deba orientarse a la demanda del mercado (lo que el mercado necesita de los profesionales).

Fuente: Elaboración propia.

Y la segunda pregunta se añade una elección sobre la formación académica, la cual es sobre una formación en investigación. Por lo cual la pregunta se presentó de la siguiente manera: ¿Crees que la formación académica debe ser más comercial (lo que demanda el mercado laboral) o de investigación? 




Gráfico 7. Elección ante el enfoque que la formación académica debe ser comercial o de investigación.

Fuente: Elaboración propia.

Estas dos preguntas en si están relacionadas debido al enfoque que se quiere expresar, pero en cuanto a las respuestas se podría considerar algo contradictorio. En primer lugar, la formación que uno recibe como estudiante siempre está sujeto a la perspectiva que tienen las autoridades en conjunto a los profesores de la universidad debido que conocen tanto el ámbito laboral como el académico. Por lo cual, en el ámbito laboral de las empresas, instituciones, centros donde se puede desempañar las respectivas profesiones. De esta forma, es algo complementario debido que los profesores consideran los cambios en la sociedad y las exigencias que la misma necesita en la formación académica que deben recibir los estudiantes, y después los estudiantes serán profesionales que irán desempeñándose de la mejor manera; y la universidad que recoge las experiencias y las exigencias de los profesionales.

Pero cuando se introdujo la segunda pregunta donde la formación comercial (lo que demanda el mercado) se ponía en elección con la formación en investigación, y la respuesta de los encuestados salió elegido la formación en investigación con un $64.9 \%$ que equivale a 649 encuestados de un total de 1000. Esto es indicado en los artículos de Medina (2015) y Rodríguez(2015) sobre la investigación que se debiera realizar en el país, y teniendo en cuenta que en los últimos años se ha incrementado los proyectos de investigación y la publicación de trabajos científicos en revistas científicas. También en muchos casos en los "workshops académicos", conferencias, jornadas académicos se pone en manifiesto que la investigación está en un alto nivel en otros países, y las experiencias obtenidas como sociedad en cuanto al crecimiento 
económico, cultural, académico y social. Y es un ejemplo a seguir debido que el Perú tiene todo lo necesario para obtener resultados notables por el bien de la sociedad académica y social.

Pero todos los posibles eventos que se puedan realizar para difundir los diferentes temas de investigación en las distintas áreas de la ciencia. En este mundo de la investigación se tiene completamente todo un sistema de protocolos, formas, medios de poder realizar investigación y de ahí, poder publicarlo en una revista científica. Esto es, que el artículo científico publicado en una revista científica es una de las pruebas más tangibles donde se pone en evidencia de una investigación científica para el mundo donde todas las personas interesadas pueden acceder a ella. Por cual, se realizó dos preguntas sobre el conocimiento de los artículos científicos y las revistas científicas. La primera pregunta es la siguiente: ¿Sabes, qué es un artículo científico (paper)? Y la segunda pregunta que le siguió fue la siguiente: ¿Sabes, cuantos niveles de revistas existen?

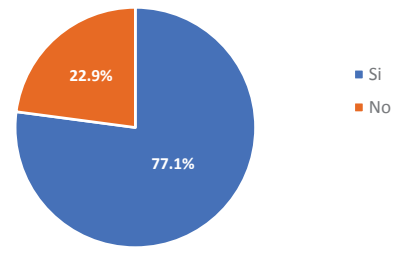

Gráfico 8. Conocimiento sobre los artículos científicos.

Fuente: Elaboración propia.

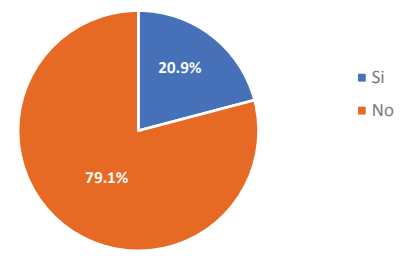

Gráfico 9. Conocimiento sobre el nivel que poseen las revistas científicas. Fuente: Elaboración propia. 
Estas dos respuestas parecieran que fueran contrapuestas, debido que el conocimiento de los artículos científicos (papers) es bastante grande $(77.1 \%)$ por mientras que el conocimiento de los niveles de las revistas científicas es bastante bajo (20.9\%), por lo cual se necesitaría una difusión más detallada sobre lo que consiste un artículo científico y sobre los niveles que poseen las revistas científicas. Esta problemática de no conocer los niveles que poseen las revistas científicas es bastante crucial para poder realizar investigación. Donde se pueden clasificar cual es de un nivel mayor, considerando la calidad y el impacto. La revista de comunicación Sociedad Española de Estudios de la Comunicación Iberoamericana (SEECI) permite tener un conocimiento sobre las revistas científicas y en el siguiente link se puede encontrar más detalles sobre el impacto que tienen las revistas científicas https://bib.us.es/comunicacion/ aprendizaje_investigacion/common/Revistasdeimpacto.doc.

Con respecto a los niveles de las revistas científicas, se clasifican en cuartiles. Existen cinco cuartiles que se les asigna a las revistas científicas. En este sentido, la CONCYTEC (Consejo Nacional de Ciencia, Tecnología e Innovación Tecnológica) ha realizado diferentes eventos para comunicar, informar, orientar, y ayudar a realizar artículos de alto impacto con una calidad internacional. Las revistas que poseen cuartiales uno hasta cuatro (Q1 - Q4) se refieren a las revistas científicas indexadas. Por mientras, que las revistas científicas que poseen cuartil cinco (Q5), son las revistas científicas en vías de indexación.

Con respecto a la publicación en las revistas científicas indexadas de cuartil uno (Q1) son consideradas, coloquialmente, como revistas top de investigación. Sin lugar a duda toda revista científica se enfoca a ser considerada en un mejor cuartil del que posee para ser considerada en el mundo de la investigación, debido que cuanto mejor sea la calificación de la revista atraerá a excelentes investigadores que quieran publicar en la revista.Teniendo en cuenta estas referencias de los artículos científicos y de las revistas científicas, se presentó la siguiente pregunta: ¿Crees que es importante la publicación de artículos de investigación durante la formación académica de pregrado? 


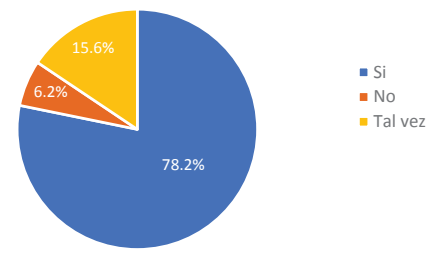

Gráfico 10. Importancia de la publicación de artículos de investigación durante la formación académica de pregrado.

Fuente: Elaboración propia.

Como se pude apreciar la gráfica, se puede considerar que los encuestados creen que es importante realizar investigación durante su formación de pregrado (78.2\%).

Desde esta perspectiva se consideró las siguientes preguntas que tienen relación con respecto a los artículos y a la publicación de los mismos en las revistas científicas. Ahora se tiene visiones sobre la directriz de la formación académica orientada a la investigación, por otro lado, actualmente se tiene etapas durante la formación académica. La primera etapa se finaliza cuando se obtiene al aprobar todos los créditos que cada carrera profesional exige para que la universidad otorgue el título de Egresado. Para luego seguir con los cumplimientos de los requisitos que exige cada facultad a sus egresados para otorgarle el diploma del grado académico de Bachiller. Y al final del proceso del bachillerato, se continúa con el cumplimiento de los requisitos exigidos por la facultad para otorgar el diploma deltítulo profesional.

Si se fomentara la investigación científica durante la formación académica de pregrado, de los alumnos en conjunto de los profesores se podría generar conocimiento e innovación en las diferentes áreas académicas. Y eso, se evidenciaría mediante la publicación de artículos científicos en adecuadas revistas científicas. Desde esta idea interesante e incentivar la investigación y la elaboración de artículos científicos, se formuló dos preguntas con respecto a la publicación de artículos científicos y a los requisitos que exige la facultad para el otorgamiento del grado académico de Bachiller y el título profesional. Teniendo estos referentes se formuló las siguientes dos preguntas a los encuestados: 
¿Estarías de acuerdo que se te exija como requisito una publicación de un artículo para obtener el grado de bachiller? ¿Estarías de acuerdo que se te exija como requisito una publicación de un artículo para obtener el título profesional?
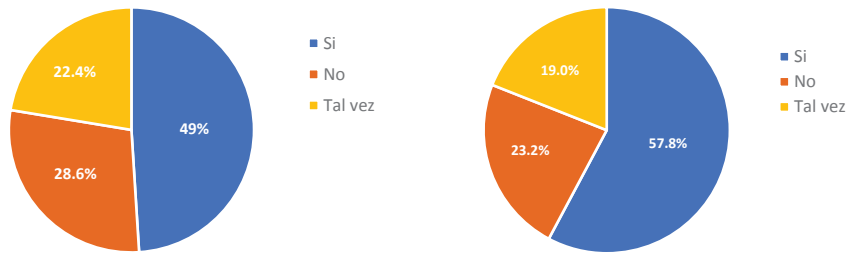

Gráfico 11. Aceptación como requisito la publicación de un artículo de para obtener el grado académico de bachiller y el título profesional.

Fuente: Elaboración propia.

Sin lugar a dudas, en cuanto a estos requisitos que actualmente no se encuentran en la ley universitaria y no se exige en las facultades como un requisito obligatorio ha tenido una considerable aceptación entre los encuestados. Para el grado de bachiller (grafica de la izquierda) se obtuvo un $49 \%$ de aceptación y con un $22.4 \%$ que se quedaron pensando en la propuesta (lo que significaría que una inclinación positiva a la propuesta). Por mientras que para el título profesional (grafica de la derecha) se incrementó la aceptación a 57.8\%, y con un 19\% que igualmente se quedaron pensando en la propuesta. No cabe lugar, que estos requisitos que son muy cuestionables y debatibles que se pongan en práctica en la actualidad pero generarían la redacción y la publicación de artículos científicos de manera que se considere una contribución adecuada para la sociedad.

No cabe duda, que en los últimos años, la investigación ha tomado bastante fuerza en las universidades de los distintos países delos diferentes continentes, debido que ha abierto brechas en creación de tecnología para el bien del propio país y con la posibilidad de generar patentes, con lo cual se podría obtener ingresos económicos y tecnológicos con otros países. Una oportunidad de trabajo conjunto entre investigadores de diferentes países donde la distancia geográfica ya no es un problema, el cual en anteriores años era un gran problema, y la consideración que los trabajos conjuntos puedan ser mostrados en diferentes piases abriendo puertas a financiamiento y generación de más proyectos de investigación. 
Ahora, con respecto a la investigación, siempre ha habido carreras profesionales que han estado más cercanas a realizar investigación y quizás otras no. Pero en verdad, tal vez esta idea equivocada deba ser rechazada para considerar que todas las carreras profesionales puedan realizar investigación, debido que existen revistas científicas de casi todas las profesiones. Solamente basta revisar las revistas internacionales para poder comprender que todas las carreras se puede realizar investigación, sobretodo la investigación interdisciplinaria que en los últimos cinco años se ha incrementado enormemente en todo el mundo, y el Perú está recién abordando con bastante impacto tanto en las áreas de la ciencia, como entre los investigadores del propio país como en colaboración de otros países. La investigación sin lugar a duda, se tornado el mayor impacto de relación sociocultural en busca de innovación tecnológica, cultural, científica y humana.

Aun en estos días, con respecto a los profesionales, se sigue pensando que la investigación solo es para algunos, la elite, el grupo pequeño destacado. Y con respecto a las carreras, se sigue pensando que las ciencias básicas, las ingenierías, y algunas ciencias sociales son las únicas donde se realiza investigación; lo cual es un error ese pensamiento, debido que el mundo ahora realiza investigaciones interdisciplinarias en conjunto de profesionales de todo el mundo mediante los grupos de investigación.

El prejuicio de la investigación de solo algunos pueden realizar, quizás sea el principal retraso en la concepción de realizar investigación. Por lo cual, se presentó la siguiente pregunta a los encuestados:

\section{¿Estarías de acuerdo que TODOS LOS PROFESIONALES deban publicar en revistas de investigación?}

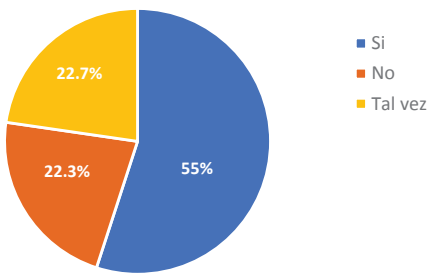

Gráfico 12. Conformidad ante la exigencia de realizar investigación de todos los profesionales y publicar artículos científicos en revistas de investigación. Fuente: Elaboración propia. 
Con respecto a esta pregunta suscito mucha controversia y cuestionamiento sobre sitodas las carreras profesionales deban investigar, pero dentro de los encuestados se obtuvo un 55\% de aceptación y un $22.3 \%$ de rechazo. Esto indica que en los últimos años de la formación académica se está tornando en una visión de investigación, o como muchos llaman una investigación comercial, países como Canadá, China, Argentina han tenido incrementos en publicaciones científicas y generación de patentes más que otros países que ya tenían cimentado la investigación como generación de riqueza, entre las cuales destaca Estados Unidos, Alemania, Japón, Brasil, entre otros, es lo que se expresó en la conferencia "De Ia investigación al artículo publicado en una revista científica de alto impacto" que fue realizado el 9 de junio de 2016 por la CONCYTEC.

No hay duda que a pocos años, casi todas las carreras tendrán el sello que deben orientarse a la investigación y a la resolución de problemas que la sociedad. También que sin considerar la idiosincrasia de las personas con respecto a realizar investigación, o mejor dicho, formalizar mediante la redacción en un artículo científico para que sea publicado en una revista viene a ser una tarea ardua. Y estoy conlleva, a ver que un poco más de la mitad de los encuestados respondieron con un respuesta afirmativa la pregunta realizada con respecto a la investigación y la calidad académica, como dos ejes que se complementarían adecuadamente; la pregunta fue la siguiente: ¿Crees que aumentado la exigencia en la investigación, se mejorará la calidad académica?



Gráfico 13. Percepción que al aumentar la investigación científica se mejorara la calidad académica.

Fuente: Elaboración propia.

Con respecto a los resultados de esta pregunta, se notó que la negativa se dio en las carreras que usualmente no realizan investigación, 
o mejor dicho, que en su carrera no se realiza investigación. Quizás de esta pregunta se realizó la última pregunta que es la cumbre de la percepción sobre la propuesta que se realiza en el presente artículo sobre realizar investigación y de publicarlo en revistas científicas.

Por lo cual, al final de esta perspectiva se realizó una pregunta que causo mucha sorpresa y dilema con respecto a una política educativa universitaria debido que quizás rompería el esquema de la formación y de la realización profesional después de obtener el título profesional hacia adelante.

La pregunta crucial de la presente encuesta con respecto a la perspectiva de investigación constante de todas las profesiones.

¿Estarías de acuerdo que de acuerdo a tu grado académico se te exija publicar en una revista científica en un tiempo determinado, y en caso contrario que no lo realices, se te quite la validez de tu respectivo grado académico, hasta que cumplas con la publicación del artículo?

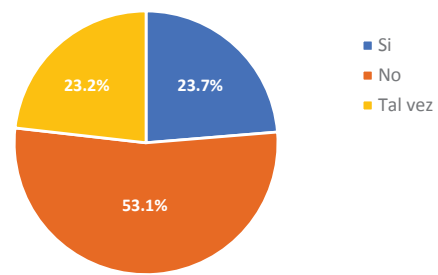

Gráfico 14. Conformidad de la exigencia de la publicación de un artículo científico en un tiempo determinado, caso contrario, se suspendería la validez del grado académico hasta el cumplimiento de la publicación del articulo.

Fuente: Elaboración propia.

Esta pregunta generó un dilema debido que cuestiona toda la percepción de la formación académica, pero sobre todo cuando uno ya es profesional (mínimo con el título profesional y el grado académico de bachiller) se dedica a ejercer su carrera en las diferentes áreas laborales de la sociedad (empresas, ministerios, instituciones, consultoras, universidades, colegios, etc.). Debido que siempre se ha dado la formación académica de la manera tradicional, la que actualmente se sigue dando, en nuestra sociedad, y este nuevo requerimiento que se podría exigir 
cambiaria rotundamente el cambio de sistema laboral, esto es debido que para mantener la validez del título profesional que es necesario para poder trabajar en todo en Perú, en caso que no se tenga el título profesional, no se te obtendría los beneficios que te brinda ser titulado, y en muchas profesiones como los médicos, abogados, ingenieros se necesitan ser colegiados y habilitados; por lo cual no tener la habilitación del colegio profesional que un requisito indispensable es el título profesional, mejor dicho que el estado peruano reconozca la validez del título profesional. Por estas razones, se podría deducir los motivos que una parte de los encuestados rechazaron esta propuesta (53.1\%) y otra parte eligieron la opción de tal vez (23.2\%) debido que no sabrían quizás las consecuencias y/o los beneficios de esta nueva reforma. Pero también hubo encuestados que eligieron esta nueva perspectiva académica y laboral (23.7\%) para una nueva forma de realizarse como profesionales.

Pero en esta pregunta muy singular contiene muchas interrogantes adicionales, debido que no solamente es la acción de publicar en una revista científica; sino también la calidad de la investigación. Y quizás ahora, la consideración de los respectivos grados académicos generen en el eje central relacionada a la calidad académica que un profesional debe realizar. Como se había mencionado anteriormente, existen cinco cuartiles que poseen las revistas científicas (considerando que el cuartil uno es la revista de más alta calidad académica y la de cuartil quinto está en vías de indexación) y estas exigen sus propios requisitos en sus propios estándares. Se tiene entendido que la revista que posee el cuartil uno (Q1) tiene muchas más exigencias y un procedimiento más detallado y minucioso (lo que no quita que otras revistas también lo exijan).

Teniendo esta perspectiva los profesionales que poseen un doctorado deberían publicar necesariamente en revistas científicas de cuartil uno (Q1) o cuartil dos (Q2), por mientras que los profesionales que poseen una maestría deberían publicar necesariamente en revistas científicas de cuartil (Q2) o cuartil tres (Q3) sin quitar el hecho que puedan publicar en una revista de cuartil uno (Q1). Finalmente, los profesionales que poseen su título profesional deberían publicar en revistas de cuartil tres (Q3) o cuartil cuatro (Q4) sin quitar el hecho que puedan publicar en una revista de cuartil uno (Q1) o cuartil dos (Q2). Con respecto a las revistas científicas de cuartil cinco (Q5) deberían orientarse más a los estudiantes que inicien 
en su formación académica orientado a la investigación y redacción de artículos científicos. No hay que olvidar que un artículo científico no se escribe por una sola persona (es muy poco visto) sino por un grupo de personas que comparten una misma visión; y más aún ahora que el mundo va tornándose en un ámbito de investigación interdisciplinario.

Otro punto de la pregunta incluye el tiempo determinado que conlleva el tipo de revista donde se piensa publicar, a mayor grado académico debería ser mayor el tiempo de requerido en un periodo estándar, teniendo en cuenta que en muchas profesiones se tiene en cierto modo mucha más facilidad de publicar que otras, se requiere muchos más recursos económicos que otras. Ante este hecho, se propuso en cierto modo que para un doctor debería tener un tiempo determinado estándar de cinco años, por mientras que el magister debería tener un tiempo determinado estándar de cuatro años y un profesional con título debería tener un tiempo determinado de tres años. Esto indicaría, que serían los tiempos que se consideraría para cumplir este requisito para seguir manteniendo la validez de su grado académico.

El tiempo parece el más idóneo considerando el grado académico y el nivel de revista que debe publicar, quizás este sea el punto de quiebre de todo profesional debe considerar para seguir manteniendo la validez del grado académico o título profesional sin considerar el tiempo que dedica a su desarrollo personal, profesional, laboral, etc. El cual fue unas de las objeciones de muchos de los encuestados, en especial de los profesionales que actualmente están trabajando en sus respectivas profesiones, por mientras que los estudiantes se presentan de acuerdo a esta perspectiva, considerando que están en la etapa formativa actualmente, considerando que con el tiempo de los cursos de pregrado van obteniendo conocimientos, experiencia, y asesoramiento por parte de sus docentes para generar artículos y poder ser publicados en revistas de investigación tanto nacionales como internacionales, siendo un complemento adecuado para el desarrollo académico y profesional de los estudiantes y una motivación para los docentes universitarios.

Presentaremos algunos comentarios ante la pregunta crucial de la encuesta realizada tanto los que apoyaron la propuesta como rechazaron 
la propuesta, ambos tipos de comentarios permiten conocer las reacciones que generaron esta nueva perspectiva.

Entre los comentarios que apoyaron la perspectiva de una investigación continua para todas las profesiones que se ejercen en el país serían las siguientes:

- Es importante, como profesionales, estar actualizados sobre nuestras carreras y a la vez también lo es estar en constante investigación para contribuir a la comunidad intelectual.

- Porque estamos preparados para hacer un artículo científico y a ese nivel debemos de llegar como se hacen en otras universidades del mundo.

- Porque con eso te vas actualizando acerca de nuevos conocimientos y nuevos descubrimientos en tu rama, no te quedas estancado en la información universitaria que se te brindo, la cual ya estará completamente desfasada para cuando termines la carrera.

- Porque con la exigencia, el estudiante universitario será capaz de realizar logros grandes, que le ayude ser un buen profesional. Y no ser uno más de los que ya hay, sino mucho mejor de los profesionales que existen o hay.

- Porque creo que la investigación es fundamental, principalmente en la formación académica, personalmente la propuesta me parece muy interesante ya que gracias a la exigencia se logra grandes cosas, y la responsabilidad es un rol que toda persona debe desarrollar.

- Sería una buena manera de impulsar la investigación y por ende estar más informado en cuanto a tu carrera y como resultado a esto tendríamos buenos profesionales.

Del mismo modo, se presentan los comentarios que rechazaron la medida propuesta.

- No, porque para realizar una buena investigación se necesita el tiempo que sea necesario para que se pueda desarrollar bien, y no a todos los profesionales les interesa el área de investigación. 
- Porque la exigencia es demasiada para una carrera comercial (administración, contabilidad, derecho, economía).

- Porque no puedes poner en cuestión tu nivel académico a la publicación de un artículo, y no tomar en cuanto el tiempo y la dedicación que le has puesto para obtener tus logros.

- Pienso que es una medida muy extrema, si en caso se aplicara a mi facultad, antes tendrían que darme una preparación para publicar algo que sea bueno y de calidad y no cualquier cosa, en ese caso sí estaría de acuerdo, pero igual yo que sepa las personas que publican en mi facultad lo hacen junto a un profesor, y si no me equivoco el profesor escoge a los alumnos de los mejores ponderados.

- No, porque dependería del tiempo disponible que tenga para poder ejecutar o redactar artículos de investigación. Y no lo veo necesario en verdad.

Finalmente, una parte de los encuestados se mostró en duda (tal vez) con respecto a la propuesta y sus comentarios fueron los siguientes.

- Si lo que se busca es incentivar la investigación, la sanción es el método menos adecuado. El estado debería proponer políticas de financiamiento a proyecto de investigación, subsidiaria investigaciones y motivar al sector privado a invertir en investigación. No presionar al profesional a hacer algo, que posiblemente lo haga por cumplir.

- Porque puede no realizarse dicha investigación por motivos endógenos, como enfermedad o accidente, y tendría que haber un sistema de apelación para la sanción, a una respectiva "comisión de justicia" y muchas cosas de esa índole, que harían las cosas caóticas y burocráticas. Estoy de acuerdo con la publicación pero debe haber otro incentivo o castigo diferente.

- Porque considero que la investigación es a elección del profesional si decide hacerlo. Con ello no me opongo a la investigación académica, sino que los que se dedican exclusivamente están más idóneos para realizarlo siempre. 
- Porque una investigación demanda de tiempo y si trabajas no te quedara tiempo para hacerlo y por ello no es justo que te quiten el grado. A menos que haya un subsidio por realizar la investigación.

- Tal vez. Porque la investigación no es obligatoria y cada profesional decide ser solo lector o investigador. Las ambiciones de cada profesional es diferente. Pero si estoy de acuerdo con la publicación de una tesis para alcanzar cada rato académico cono en el sistema actual.

De esta forma, terminamos la sección sobre la percepción de esta propuesta de investigación continua realizada a estudiantes y profesionales. Las diferentes preguntas realizadas han ido mostrando el apoyo y el rechazo, y la duda entre apoyar o rechazar la propuesta, debido que cambiaría rotundamente la formación académica de pregrado, y el propio desarrollo profesional y laboral después de obtener el título profesional. Sin lugar a duda, estas interrogantes nos permitieron conocer sobre el conocimiento que se tiene sobre el mundo de la investigación que en nuestro país está en un nivel pobre a comparación de otros países tanto en Sudamérica como en el exterior. Hay muchas interrogantes y dudas sobre esta perspectiva algo radical con respecto a la investigación, pero también hay iniciativas que actualmente se dan en nuestro país, especialmente generado por la CONCYTEC tanto como financiamiento como fomentación a nivel empresarial e institucional.

Para complementar la perspectiva presentada con las actividades, proyectos, perspectivas que se realizan actualmente mediante la CONCYTEC.Sus iniciativas que han tenido en los últimos años, han generado financiamiento e impulsamientoen proyectos de investigación con más énfasis desde la directiva de la doctora Gisela Orjeda a la actualidad. Y elDirectorio Nacional de Investigadores e Innovadores (DINA) que es una base de datos que registra las hojas de vida los profesionales peruanos que realizan actividades de ciencia, tecnología e innovación (CTI), tanto en el Perú como en el extranjero. Y que también contribuye a dar visibilidad a la labor de los investigadores e innovadores peruanos, así como a vincularlos con sus pares para que puedan generar múltiples oportunidades de potenciar sus redes de colaboración científica. 
Finalmente, es importante mencionar que el sitio web de la DINA,localizada en el siguiente link https://sites.google.com/a/concytec. gob.pe/manual-dina/introduccion, expresa de si misma que el Directorio Nacional de Investigadores e Innovadores se vincula con un módulo georeferencial denominado GeoCONCYTEC que permite visualizar en tiempo realla distribución de investigadores en el Perú, tanto por ubicación geográfica, género, grado académico e institución de pertenencia.

Y terminaremos este apartado del DINA, con su frase más representativa:

\section{La inscripción en DINA es gratuita, voluntario y de acceso público. Te invitamos a conocer DINA y registrarte.}

Y si se quiere conocer más de la formalización sobre la investigación que el DINA va organizando, se presenta el REGINA que es el Registro Nacional de Investigadores en Ciencia y Tecnología. Siendo el registro de personas naturales que poseen capacidades establecidas de acuerdo a una calificación, para realizar labores de investigación científica y/o desarrollo tecnológico. Este registro fue formalizado con la aprobación de la RESOLUCIÓN DE PRESIDENCIA № 184 -2015-CONCYTEC-P: donde se aprueba el "Reglamento de Calificación y Registro de Investigadores en Ciencia y Tecnología del Sistema Nacional de Ciencia, Tecnología e Innovación Tecnológica - SINACYT". Es lo que manifiesta en su propia página web del CONCYTEC y relacionado con el link https://sites.google. com/a/concytec.gob.pe/manual-dina/introduccion.

Sin lugar a duda, la investigación científica ha generado un gigantesco "boom" en la formación académica tanto de pregrado y con mayor énfasis en posgrado. Seguir redactando sobre los detalles de la investigación en el contexto peruano es bastante amplio y también bastante complicado por las normativas internacionales que recién el Perú está comenzando a difundir de una manera más integral, el cual lo realiza la CONCYTEC mediante seminarios, conferencias, encuentros, etc. Para dar a conocer los beneficios que brinda realizar investigación en nuestro país. 


\section{Conclusiones}

En verdad, realizar el presente trabajo de investigación sobre una perspectiva donde se ha considerado prácticamente radical en los comentarios por la sanción cuando no se realiza investigación en un tiempo determinado que sea adecuado a las exigencias que demande las áreas de la ciencia ha sido bastante interesante. Esto a mi parecer, quizás porque ante las nuevas tendencias de conseguir información tanto nacional como internacional mediante el internet, donde se puede conseguir libros electrónicos, artículos científicos, apuntes de clases, etc. Prácticamente el torno científico se ha tornado en una interacción virtual que rompa las barreras de la distancia geográfica.

De todo lo expuesto, se podría obtener algunas conclusiones que estuvieran abiertas a discusión, pero brindan una directriz que se podría considerar para mejorar la formación académica que en los últimos años en Sudamérica está innovando más que los otros países desarrollados donde la investigación ya está formalizada y enfocada según sus propias necesidades que necesitan resolver. Sin lugar a duda, se tiene que mejorar en el día a día en la preparación, formación y desenvolvimiento de cada estudiante para poder lograr profesionales de alta calidad académica, humanística y científica.

- Laleyuniversitariabrindaincentivosalos docentesuniversitarios, pero no a los estudiantes de una manera directa. Por otro lado, la CONCYTEC promueve la presentación y el financiamiento de proyectos de investigación mediante concursos abiertos hacia las universidades.

- En los últimos años, la investigación científica se ha tornado mediante grupos multidisciplinarios donde se complementa las diferentes áreas de la ciencia para resolver un problema de investigación.

- El Perú se encuentra en una época donde se está invirtiendo y fomentando la investigación en diversos proyectos de investigación más que años anteriores. Y con el DINA va presentando sus diversos profesionales que se dedican a la investigación, y más aún con el REGINA. 
- La tendencia de realizar investigación durante la formación académica en pregrado y la redacción de artículos científicos ha tenido una buena acogida entre los encuestados, de preferencia los que actualmente son estudiantes.

- Los profesionales han presentado algunas críticas y comentarios negativas ante la nueva perspectiva de investigación constante debido que ellos no han sido formados para realizar investigación y poder redactarlos en un artículo científico.

- Los estudiantes han presentado comentarios, críticas y observaciones positivas que permiten una buena apertura a la nueva perspectiva de investigación. Donde realizar investigación desde su formación académica, con lo cual podrían redactar artículos científicos y poder publicarlos en revistas científicas abriéndose ante el mundo científico que ahora es más competitivo que nunca.

\section{Referencias Bibliográficas}

Aldana de Becerra, Gloria Marlen \& Joya Ramírez, Nohora Stella. (2011). Actitudes hacia la Investigación Científica en Docentes de Metodología de la Investigación. Fundación Universitaria del Área Andina. Tabula Rasa. Bogotá - Colombia.

Alcántara, Carlos \& Almeida, Andrés. (17 de octubre de 2017). Descubre los resultados del Ranking de Universidades Peruanas 2017. Revista América Economía, sección Reportajes Educación. Recuperado de https://mba. americaeconomia.com/articulos/reportajes/descubre-los-resultados-delranking-de-universidades-peruanas-2017

La investigación histórica del «tiempo presente» en Alemania WALTHER L. BERNECKER Universidad de Erlangen-Númberg

Barahona Migueles, Jeileen \&Medina, Edwin Medina. (2015). Percepción de los docentes y estudiantes hacia el proceso enseñanza-aprendizaje de la investigación en la Facultad de Ingeniería, Ciudad Universitaria, 2014. Revista Portal de Ciencias, No. 8, junio 2015.

Bernecker, Walther. (1998). La investigaciónhistórica del tiempo presente en Alemania. Cuadernos de Historia contemporánea, numero 20,83-98 Universidad de Erlangen-Númberg. 
Coraggio,José Luís.(1996). Renovación universitaria y pedagógica dela enseñanza superior. Exposición en el Seminario sobre "Las Nuevas Universidades a finales del siglo XX". Universidad Nacional de General Sarmiento, San Miguel Argentina.

Desantes-Guanter, José María y José López. 1996. Teoría y Técnica de la Investigación científica. Madrid: Síntesis.

Guerra-García, Roger. (1979). La investigación científica en el Perú en los años veinte. Revista de la Universidad Católica.

Figueroa, Adolfo. (2008). Nuestro Mundo Social: Introducción a la Ciencia Económica. Fondo Editorial PUCP. 292 páginas.

Medina Tovar, Sandro. (22 de julio de 2015). En el Perú se producen pocos artículos científicos. El Comercio. Recuperado de https://elcomercio.pe/ tecnologia/ciencias/peru-producen-articulos-cientificos-182496

Mejía, Mario. (28 de junio de 2014). Nueva Ley Universitaria: aciertos, errores y omisiones. El Comercio. Recuperado de https://elcomercio.pe/politica/ actualidad/nueva-ley-universitaria-aciertos-errores-omisiones-374622

Ministerio de Educación. (2014). Ley Universitaria, ley $N^{\circ} 30220$. Ministerio de Educación. Recuperado de http://www.minedu.gob.pe/reforma-universitaria/ pdf/ley_universitaria.pdf

Oficina Central de Admisión (2016). Catálogo de carreras profesionales UNMSM. Universidad Nacional Mayor de San Marcos. Recuperado de http://www. grupopitagoras.com/assets/catalogoadmision20171.pdf

Redacción CONCYTEC. (21 de julio de 2016). CONCYTEC difunde oportunidades de financiamiento de proyectos de investigación mediante programa Horizonte 2020. Seminario "Horizonte 2020: Investigación, Desarrollo e Innovación”. CONCYTEC. Recuperado de https://portal.concytec.gob.pe/ index.php/noticias/807-concytec-difunde-oportunidades-de-financiamientode-proyectos-de-investigacion-mediante-programa-horizonte-2020

Redacción EC. (30 de abril de 2014). ¿Cómo avanza la producción científica en el Perú? El comercio. Recuperado de https://elcomercio.pe/lima/avanzaproduccion-cientifica-peru-315347

Redacción Sociedad Expo Sociedad (14 de noviembre de 2017). Estas son las mejores 15 universidades del Perú. La República. Recuperado de http:// larepublica.pe/sociedad/1145120-estas-son-las-mejores-15-universidades-delperu 
Redacción Gestión. (27 de febrero de 2014). Universidades peruanas quedan fuera del Top 100 de producción científica en Iberoamérica. Gestión. Recuperado de https://gestion.pe/tendencias/universidades-peruanas-quedan-fuera-top100-produccion-cientifica-iberoamerica-5135

Redaccion Nupex. (2015). La ciencia y la segunda guerra mundial. Nupex, Nuclear Physics Experience. Recuperado de http://nupex.eu/index. php?g=textcontent/nuclearhistory/scienceandworldwar\&lang $=e s$

Redacción Portal Alemania. (24 de octubre de 2016). Investigar en Alemania - ¿un paraíso para los investigadores?Portal Alemania: portal de noticias e información. Recuperado de http://www.portalalemania.com/estudiaren-alemania/2016/10/24/investigar-en-alemania-un-paraiso-parainvestigadores.html

RodriguezRodriguez, Michel. (2015). El mundo de las publicaciones científicas. Educación Global 19. Indd 117. Universidad de Guadalajara.

Revista de Comunicación de la SEECI. (2010). Índice de Calidad de Revistas científicas. Sociedad Española de Estudios de la Comunicación Iberoamericana (SEECI). Clave Identificativa A/027866/09. Recuperado de http://www.seeci.net/congresochile/pdfs/indices.pdf

CONCYTEC. Manual de uso del DINA. Consejo Nacional de Ciencia, Tecnología e Innovación Tecnológica. Recuperado de https://sites.google.com/a/concytec. gob.pe/manual-dina/introduccion

Universidade da Coruña. (2017). Consultar los índices de calidad de revistas científicas. Biblioteca Universitaria - Servicios - Apoyo a la Investigación. https://www.udc.es/biblioteca/servizos/apoio_investigacion/servizos_apoio/ guias/consultar_indices.html?language $=e s$ 